\title{
Mosaic structure in the spines of Holopneustes porossisimus
}

\author{
Klemens Kelm*,I, Andreas Goetz ${ }^{\mathrm{II}}$, Angelika Sehrbrock ${ }^{\mathrm{III}}$, Stephan Irsen ${ }^{\mathrm{III}}$, Ramona Hoffmann ${ }^{\mathrm{IV}}$, \\ Wolfgang W. Schmahl ${ }^{\mathrm{II}}$ and Erika Griesshaber ${ }^{\mathrm{II}}$ \\ I German Aerospace Centre (DLR), Institute of Materials Research, Linder Höhe, 51147 Köln-Porz, Germany \\ II Department of Earth and Environmental Sciences, Applied Crystallography and Materials Science, Theresienstr. 41, 80333 München, Germany \\ ${ }^{\text {III }}$ Research Centre caesar, Ludwig-Erhard Allee 2, 53175 Bonn, Germany \\ IV Department Chemie, NIM \& CeNS, Ludwig-Maximilians-Universität München, Butenandtstr. 11, 81377 München, Germany
}

Received March 27, 2012; accepted August 19, 2012

Published online: October 1, 2012

\section{Powder structure analysis / X-ray diffraction / Tetrazoles / Microstrains}

\begin{abstract}
Sea urchin spines of Holopneustes porossisimus are porous single crystals, with the pores being filled with a material rich in carbon, silicon, fluorine and sodium. The magnesian calcite constituting the spine is highly strained. Even though the spines appear to be single crystalline on a macroscopic scale, the calcitic material exhibits an extended defect network. We find dislocations as well as rotational and other, not yet identified boundaries. We also observe within spine calcite a patterned distribution of sulphur. Both distributions, that of the defect network and that of sulphur resemble in their pattern to each other and have a similar mesh size of $50 \mathrm{~nm}$. We conclude from these observations that they arise from the growth process of the spine and account for the mosaicity within the spine single crystals.
\end{abstract}

\section{Introduction}

It is common that organisms crystallize minerals to form a skeleton. Some of the most outstanding ones are sea urchins, which form a skeleton consisting of five plates forming the body and numerous spines. The spines contain a base (the connection between spine and the body of the animal, the corona) and a shaft portion. The shaft is formed by several septa or wedges that consist of massive magnesian calcite. In the spine the wedges are aligned to each other in a concentric manner. They form about $2 / 3$ of the radius of the spine and get thicker towards the outer rim of the spine. Each wedge spreads over the entire length of the complete shaft. The wedges are separated from each other by a highly porous calcite, the stereom that is also present in the center of the spine (Raupp, 1966), (Fig. 1). Even though sea urchin spines have been subject to research for more than 100 years, their high

\footnotetext{
* Correspondence author (e-mail: Klemens.Kelm@dlr.de)
}

degree of crystalline perfection is not fully understood yet.

Magnesian calcite is the constituting mineral of the spines. The magnesium content varies within a few $100 \mu \mathrm{m}$ (Moureau et al., 2010). Organic material $(0.1-0.15 \%)$ is intercalated inside the mineral matrix (Weiner, 1985). Under polarized light, the spines behave like single crystals (von Ebner, 1887, Schmidt, 1924, Schmidt, 1930). Standard Electron Backscattered Diffraction (EBSD) with its angular resolution of about $0.3^{\circ}$ leads to the same result (Moureaux et al., 2010).

However, small misorientations within the spines have been determined by the analysis of coherence length in synchrotron scattering (Berman, et al. 1990, Aizenberg et al., 1997) and rocking curve experiments (Berman, et al. 1990, Tsipursky and Buseck, 1993, Goetz et al. 2010). These misorientations have been attributed to a tilt of the wedges within the spine determined with high angular resolution EBSD (Goetz et al. 2010).

While the misorientation of the wedges appears on a level of hundreds of microns, the presence of a much finer additional mosaic structure has been suggested due to the nanometer sized, non-crystallographic structure of the fracture surface of the spines (Fig. 1c). This structure spans on a lateral scale of about 50-100 nm (Nissen, 1969, Towe, 1967, O’Neill, 1981, Oaki and Imai, 2006, Oaki et al., 2006).

There have been several attempts to reveal such a microstructure by transmission electron microscopy (TEM). The selected area electron diffraction (SAED) patterns appear to be recorded from perfect single-crystals preventing this approach from being successful (Towe, 1967, Travis, 1970, Blake, Peacor and Allard, 1984, Tsipursky and Buseck, 1993, Su, Kamat and Heuer, 2000, Imai et al., 2006, Sethmann and Wörheide, 2008). Nevertheless, Imai et al. (2006) concluded from crushed samples that an nmsized mosaic structure is present within sea urchin spine calcite. Su, Kamat and Heuer (2000) observed small inclusions with a size of about $\sim 80 \mathrm{~nm}$ within the spines, but no building faults like dislocations. Blake, Peacor and Allard (1984) as well as Tsipursky and Buseck (1993) observed semi-coherent boundaries by high-resolution 

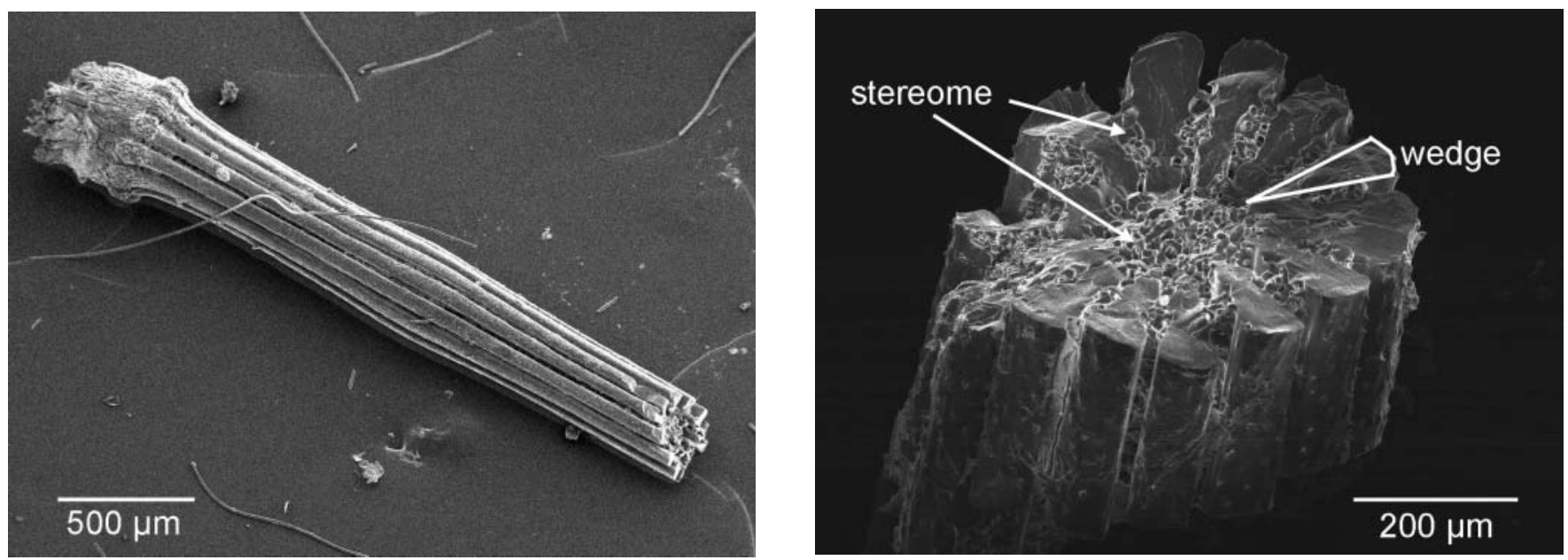

(a)
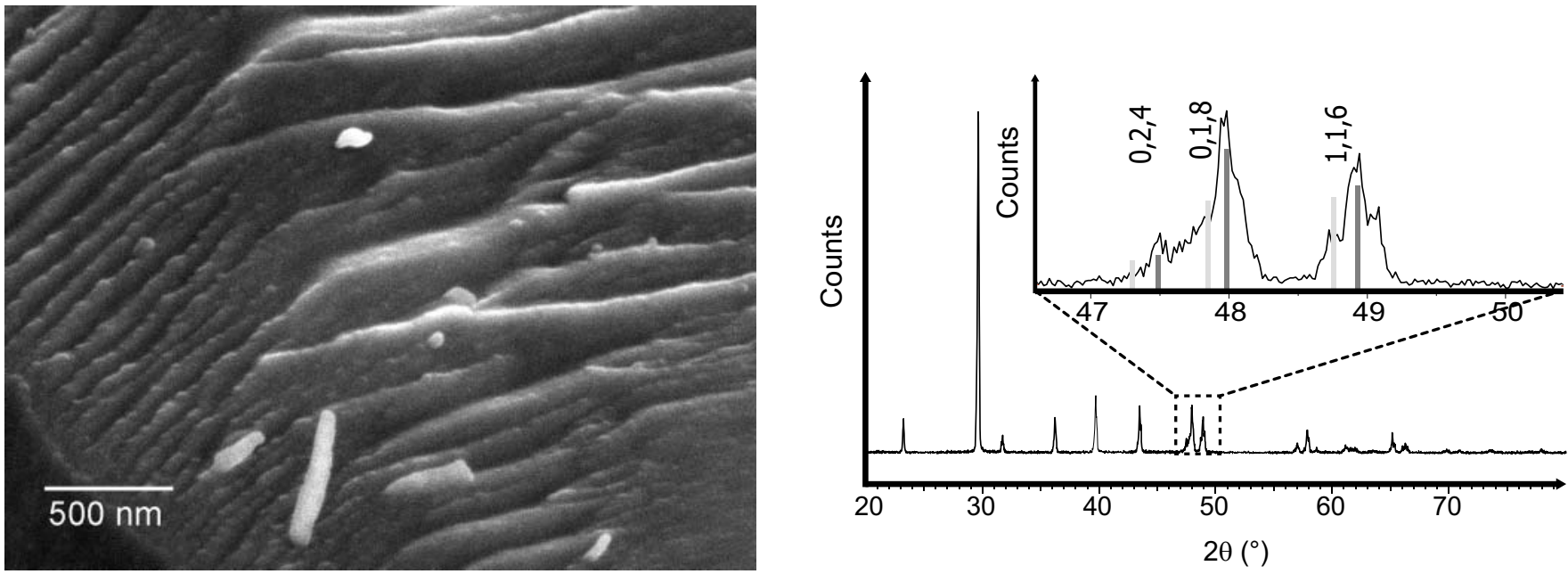

(C)

(d)

Fig. 1. SEM secondary electron image of a transversally broken spine. (a) Complete spine with tip broken off. (b) Transversally broken spine. Note the wedges and the stereome. (c) High magnification micrograph showing the nanopatterend crack structure. (d) X-ray diffraction pattern of several powdered spines. The insert shows the observed peak splitting.

TEM. Pores within the spine as well as dislocation-like lines were observed by Kelm et al. (2011). Here, we report TEM investigation, which give further insight into the presence as well as the nature of the nanometer sized mosaic structure of the spine of the sea urchin Holopneustes porossisimus.

We investigated the spine's microstructure to understand the interplay of the 'bottom up' crystallization process as reported by Politi et al. (2004) and the source of observed diffraction effects as lattice defects and integral or local misorientations.

\section{Experimental}

The spines investigated were taken from a museum specimen of the recent sea urchin Holopneustes porossisimus (Zoologische Staatssammlung München, Reg.-Nr. 20060657, collected in 1982 at Port Phillip Bay, Victoria, Australia). Details on the habitat can be found in Hutchinson, Hunt and Morris, 2010. This species was chosen because its spines fit well to the requirements of sample preparation.
They are small, about $3-5 \mathrm{~mm}$ in length and about $0.5-$ $1.5 \mathrm{~mm}$ in diameter. For TEM sample preparation, a part of the shaft of a spine, $1.5 \mathrm{~mm}$ in length and $0.5 \mathrm{~m}$ in diameter was cut off. It was clamped into a slotted titanium ring (Baltic, Germany). To fill up the highly porous structure of the spine, the whole assembly of the ring with the spine was embedded in epoxy resin (Gatan, USA). The sample was ground from both sides to form a longitudinal section with a thickness of $100 \mu \mathrm{m}$. Dimple grinding and ion beam etching with $\mathrm{Ar}^{+}$-ions at $4-2 \mathrm{KeV}$ and 2-1 mA beam current at $7^{\circ}$ incidence angle (Bal-Tec RES 101, Lichtenstein) resulted in a hole with electron transparent edges. These edges covered the wedges as well as the mineralized part of the stereom. Special care was taken to prevent external stresses during preparation. The acceleration voltage and the beam current were reduced from $4 \mathrm{keV}$ to $2 \mathrm{keV}$ and from $2 \mathrm{~mA}$ and $1 \mathrm{~mA}$, respectively during the preparation progress to prevent beam damage of the specimen.

Additionally, a second spine was ground in an agate mortar and dispersed in ethanol. A drop of the dispersion was dried on a holey carbon film (Quantifoil, Germany). 
TEM investigations were performed with a Philips Tecnai F30 microscope (Philips, The Netherlands) operating at $300 \mathrm{KeV}$. The microscope is equipped with a Gatan 794 CCD Camera (Gatan, USA) and a High Angle Annular Dark-Field (HAADF) detector (Fischione, USA) for scanning transmission electron microscopy (STEM). Furthermore, for Energy Dispersive Electron Spectroscopy (EDX), a windowless silicon drift detector (Apollo XLT, EDAX, USA) is installed. Care was taken to avoid high electron doses and beam heating of the samples during the investigation to prevent beam damage or phase decomposition.

Scanning electron micrographs were collected with a JSM 6500F scanning electron microscope (JEOL, Japan).

XRD measurements were performed on spines from which the base was removed. The ground powder was dispersed on a silicon single crystal holder exhibiting no reflections in the applied angular range $\left(20^{\circ}-80^{\circ} 2 \theta\right)$. The diffractometer (Bragg-Brentano geometry, Siemens D5000, Germany) was equipped with a secondary graphite monochromator and a $\mathrm{Cu} \mathrm{X}$-ray tube.

\section{Results}

\subsection{Morphology X-ray and electron diffraction}

In the X-ray diffraction pattern of ground spines, only magnesian calcite was detectable (Fig. 1d). A shoulder at the low angel side of most diffraction peaks, as illustrated for the 116 reflection (Fig. 1d, insert) indicated the presence of at least two magnesian calcite phases of different composition. Rietveld refinement assuming two phases resulted in $80 \%\left(\mathrm{Ca}_{0.92} \mathrm{Mg}_{0.08}\right) \mathrm{CO}_{3}\left(a_{0}=0.495725(24) \mathrm{nm}\right.$, $\left.c_{0}=1.69135(10) \mathrm{nm}, \quad V=359.967(41) \AA^{3}\right)$ and $20 \%$ $\left(\mathrm{Ca}_{0.96} \mathrm{Mg}_{0.04}\right) \mathrm{CO}_{3}\left(a_{0}=0.497301(44) \mathrm{nm}, c_{0}=1.69852(24)\right.$ $\left.\mathrm{nm}, V=363.782(82) \AA^{3}\right)$. Compositions were determined from the refined lattice parameters according to the method of Titschack, Goetz-Neunhoeffer and Neubauer, 2011.

All selected area electron diffraction (SAED) patterns showed only sharp diffraction spots of magnesian calcite, as shown in Fig. 2d. Beam damage in the TEM as well as beam damage due to $\mathrm{Ar}^{+}$-ion beam etching results in the formation of calcium oxide, easy detectable by its rings pattern overlaid on the calcite spot pattern in selected area electron diffraction. Such patterns were not observed during the investigation, indicating that no sever beam damage had occurred.

\subsection{Abundance of pores and pore morphology}

On SEM micrographs the wedges of the spines appear to consist of massive calcite connected together by the stereom, which forms a three-dimensional calcite meshwork. In Holopneustes porossisimus, the size of the interconnected pores within the stereom is about $10 \mu \mathrm{m}$ (Fig. 1a, b).

The wedges as well as the stereom are highly interspersed with closed pores (Figs. 2a, b and 6). Some of the

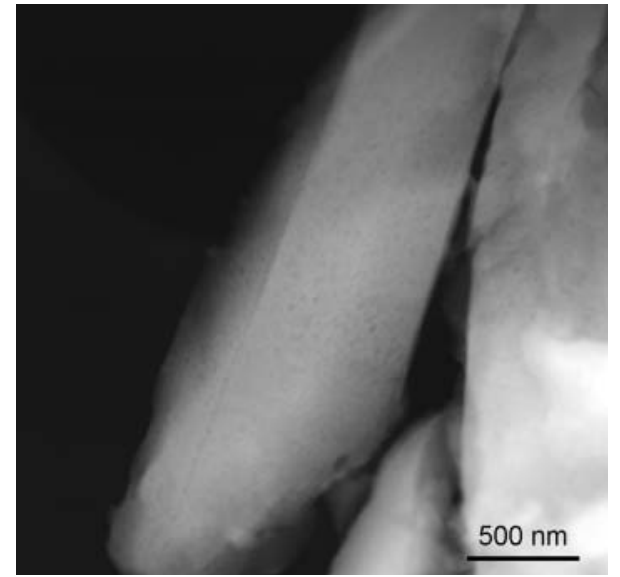

(a)
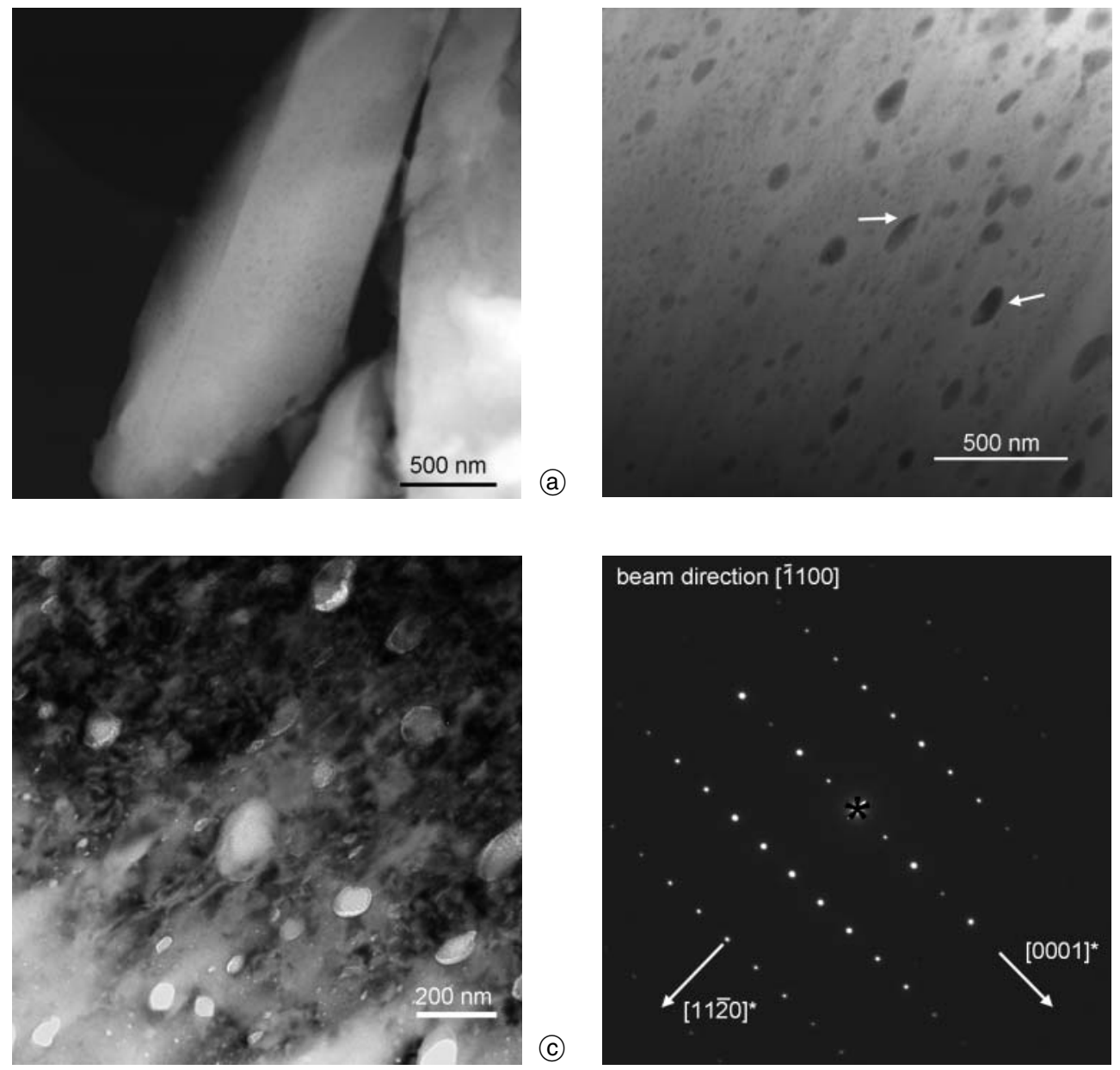

(C)

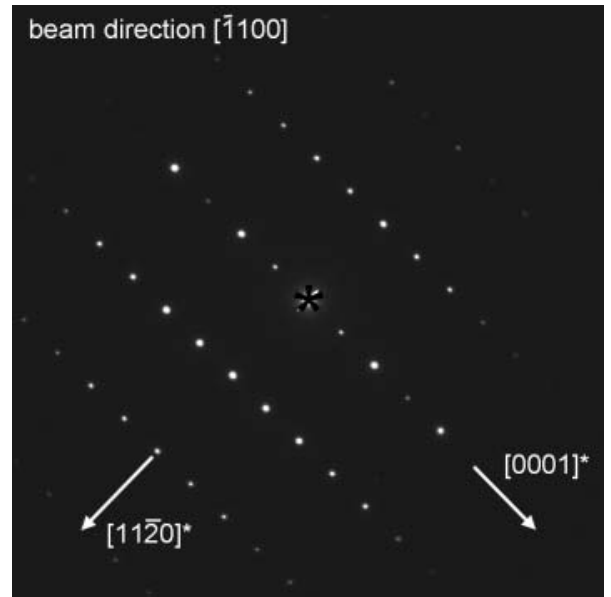

Fig. 2. HAADF STEM image of a crunched spine (a) and a longitudinal cross-section of a spine of Holopneustes porossisimus. Arrows mark pointed pores (b). Bright field TEM image (c) and corresponding SAED pattern (d) of a spine. In the applied viewing direction, pores appear with low or very high aspect ratio. 


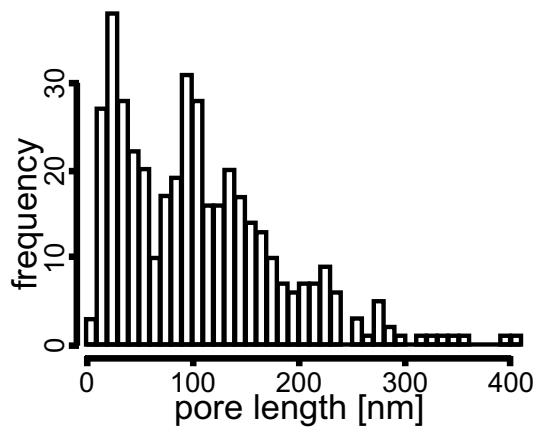

Fig. 3. Pore size distribution in the mineralized part of the spines.

pores have been opened up by the preparation procedure and appear as holes in the thin rim of the sample or exhibit grainy contrast due to redeposited material (Fig. 2c). However, on HAADF STEM images, their distribution, size and morphology is clearly visible (Figs. 2b and 6). The pore's size ranges from $5 \mathrm{~nm}$ to $400 \mathrm{~nm}$. We calculated a pore size distribution by counting the pores and measuring their size on a representative selection of our micrographs. The distribution is bimodal with maxima at $30 \mathrm{~nm}$ and $100 \mathrm{~nm}$ (Fig. 3).

The pores appear with oval shape on the images taken with the sample orientated to an arbitrary, high-indexed

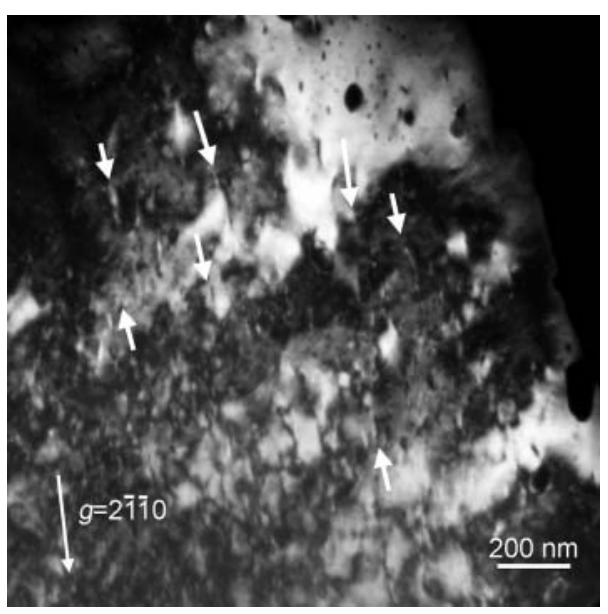

(a)

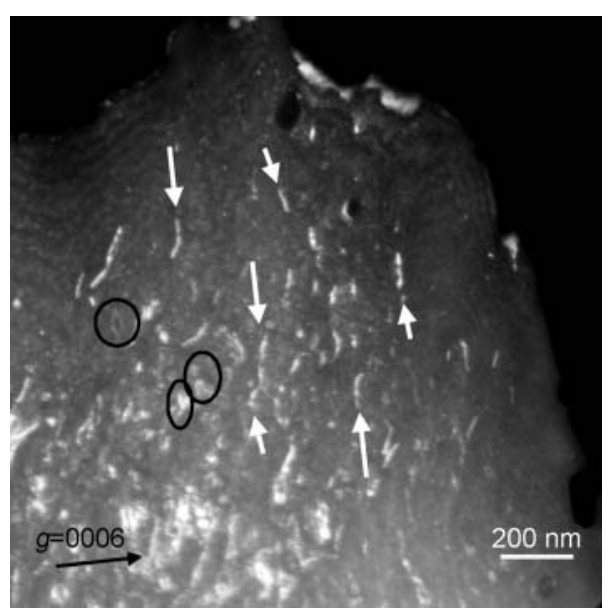

(b)

Fig. 4. Two beam dark field images of the same region of the spine recorded using the indicated operating reflection. White arrows indicate dislocations. Pores exhibiting peanut-shaped contrast encircled. crystallographic orientation. The long axes of the pores are aligned with respect to each other. Some of the oval pores also exhibit pointed edges in the long axis (Fig. 2b). On images taken with the beam parallel to the $\langle 1 \overline{1} 00\rangle$ direction of the calcite, some pores appear with a much lower aspect ratio. Even nearly round pores are present (Fig. 2c). Additionally, numerous slim pores with a high aspect ratio can be observed on such images (Fig. 2c, d). Thus, the pores span in the $a-c$-planes of the calcite.

It is worth noting that we did not observe any difference between the materials of the wedges and the stereom in our images.

\subsection{Diffraction contrast experiments}

Strong diffraction contrast is visible throughout the samples. If two-beam conditions are applied, we can distinguish several prominent features.

There are linear features running roughly parallel to the $a$-axis of the calcite (Figs. 4 and 5, white arrows). Such linear features appear if dislocations are present. Dislocations should vanish if the operating lattice vector $g$ used for imaging and the burgers vector $b$ of the dislocation are perpendicular to each other (Hirsch et al., 1965). Unfortu-
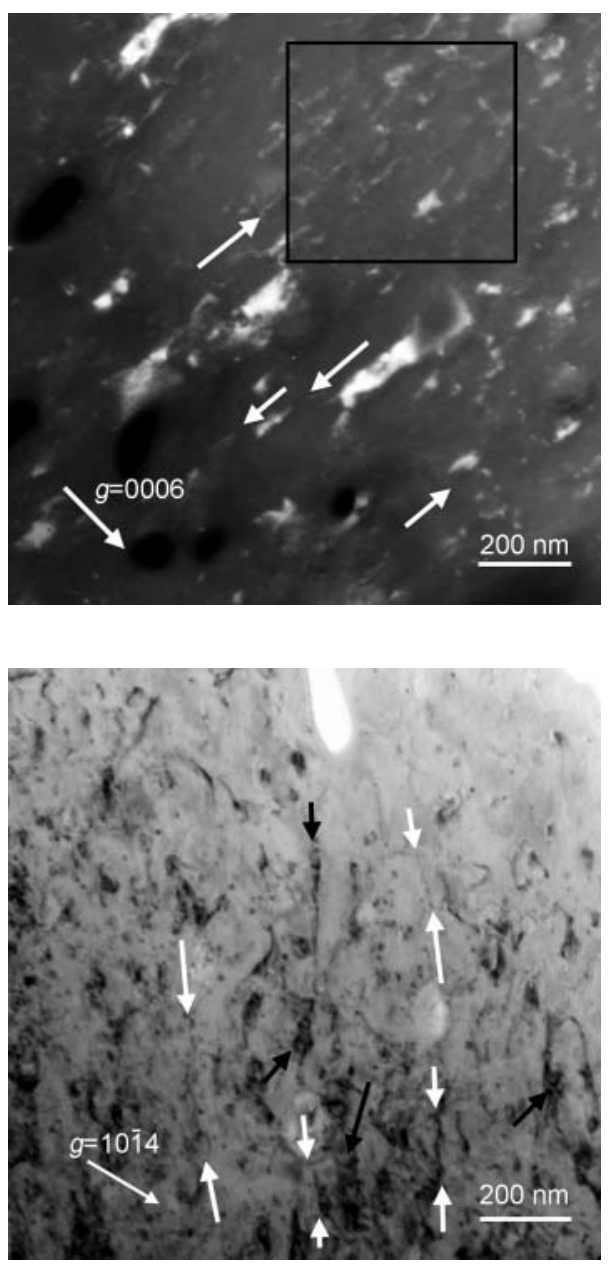

(b)

Fig. 5. Two beam dark field image (a) and bright field image (b) of different regions of the spine recorded using the indicated operating reflection. White arrows mark the dislocation lines; black arrows mark misorientation boundaries exhibiting fringe contrast. The black square indicates area with a crystal fault network. 
nately, we were not able to find such conditions where the contrast of the lines vanishes completely, but we observed much lower contrast of the lines if $g=(0006)$ (Fig. 4b) or $g=(01 \overline{1} 8)$ were chosen as operating reflections. Assuming the observed contrast to be residual contrast, a burgers vector of $b=1 / 3[2 \overline{1} \overline{1} 0]$ is present. Residual contrast is common for deformed inorganic calcite (Barber et al., 2007).

Another feature is a very pronounced diffraction contrast (Fig. 4a). This contrast is observed for all operating reflections except $g=(0006 n)$. Such a contrast arises from lattice deformations; thus the samples are in an extremely stressed state.

In images exhibiting less pronounced contrasts, occasionally additional bright areas can be observed in dark field micrographs (Fig. 5a). Under favorable imaging conditions, these contrasts develop to systems of a few faint parallel lines, indicating boundaries (Fig. 5b, black arrows). As all observed diffraction patterns did not indicate twins or polycrystalline material, these features have to be stacking faults or rotational boundaries giving rise to $\delta$-fringes. Also, a faint network of interconnected bright lines is visible exhibiting a mesh wide of about $50 \mathrm{~nm}$ (Fig. 5a).

\subsection{EDX measurements}

The EDX spectra of the material revealed calcium, magnesium, oxygen and carbon as the main constituting elements of the spine. Furthermore, small portions of strontium, chlorine, sulfur, silicon, sodium and fluorine were detected. Quantification was carried out by the standardless Cliff-Lorimer technique. No attempt was made to quantify oxygen and carbon, as the X-rays of these elements suffer strong absorption inside the sample. Also, the sodium content has to be regarded as a lower limit since sodium loss was observed during spectrum acquisition. The typical error of EDX analysis in TEM is about 1 at.\% due to systematic errors in background removal and $k$-factor estimation, meaning that the content of most of the additional elements observed is around the lower limit for reliable quantification. A representative quantitative analysis is given in Table 1.

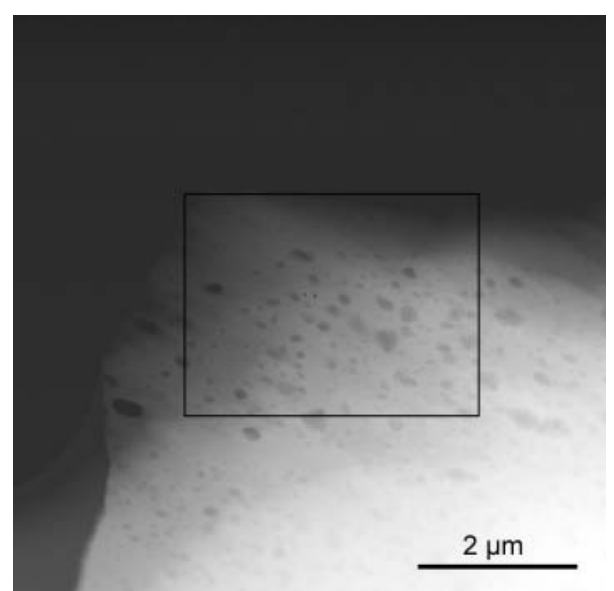

Fig. 6. HAADF STEM image of the spine. The region used for EDX mapping is marked with the black rectangle.
Table 1. Quantitative EDX results of a representative sample area.

\begin{tabular}{lcclcl}
\hline & $\begin{array}{l}\text { Weight } \\
\%\end{array}$ & $\begin{array}{l}\text { Atomic } \\
\%\end{array}$ & $\begin{array}{l}\text { KAB } \\
\text { Factor }\end{array}$ & Net Int. & $\begin{array}{l}\text { Net Error } \\
\%\end{array}$ \\
\hline F K & 0.2 & 0.5 & 2.26 & 3.86 & 1.69 \\
Na K & 0.9 & 1.6 & 0.96 & 35.22 & 0.29 \\
Mg K & 1.4 & 2.3 & 0.98 & 52.6 & 0.21 \\
Si K & 0.2 & 0.2 & 1 & 5.58 & 2.11 \\
Sr L & 1 & 0.5 & 3.35 & 10.69 & 1.49 \\
S K & 0.8 & 1 & 0.99 & 28.38 & 0.35 \\
Cl K & 0.3 & 0.4 & 1.02 & 11.41 & 1.18 \\
Ca K & 95.1 & 93.6 & 1.16 & 2953.77 & 0.02 \\
\hline
\end{tabular}
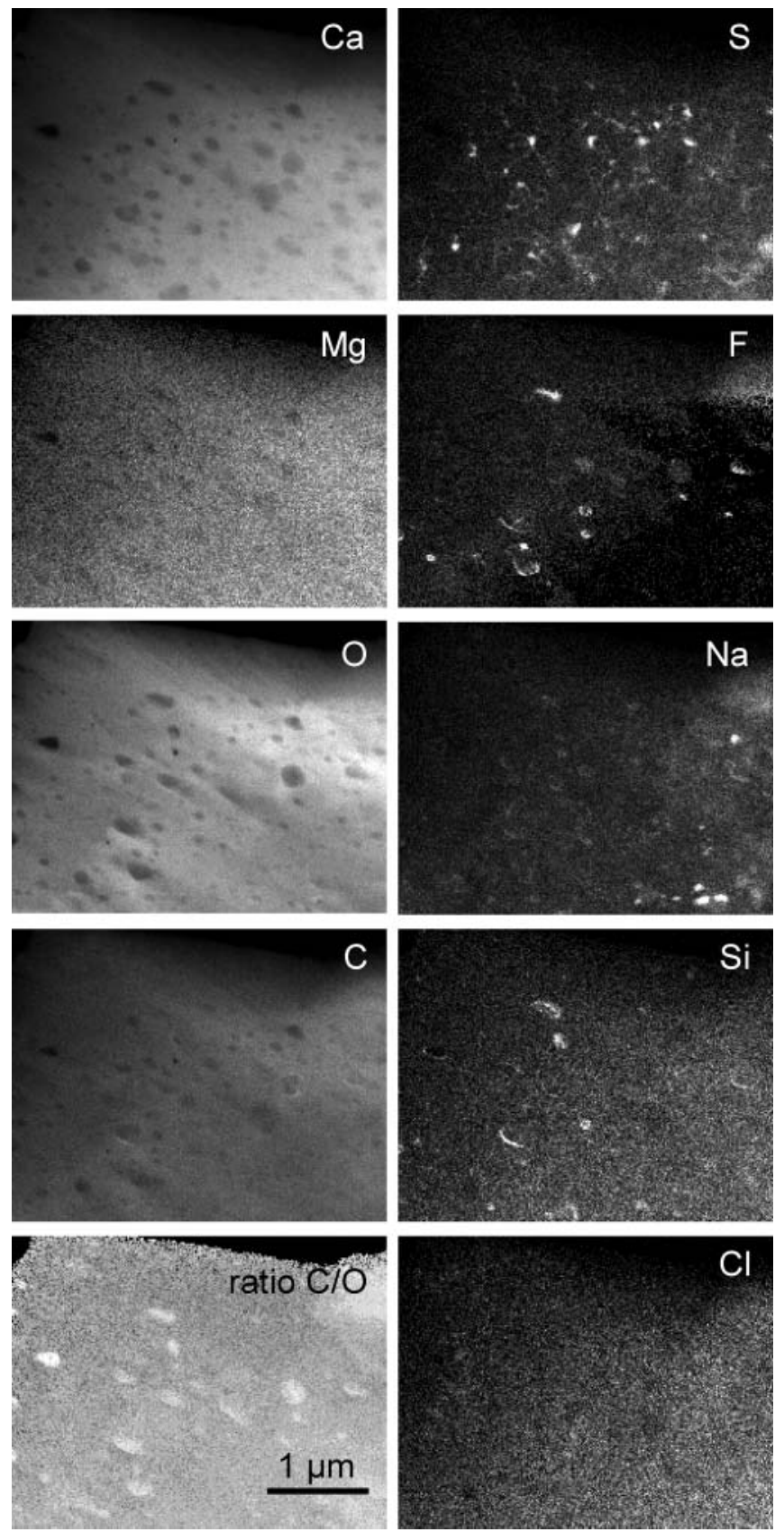

Fig. 7. EDX maps (gross counts, K-lines) of the spine. The image in the lower left corner is the ratio map produced by dividing the carbon and oxygen maps. Original map size $200 \times 256$ pixels. 
Nevertheless the EDX mapping in TEM often is hampered by counting statistics, it was possible to collect elemental maps of the main elements. Local enrichment allowed also to record reliable maps of most of the trace elements. Only the strontium signal was too low to record a reliable map containing anything else than noise (not shown). The area used for element mapping is given in Fig. 6.

The calcium and oxygen maps reveal the presence of the pores inside the calcite matrix and resemble the HAADF image as shown in Fig. 6. The maps of magnesium and chlorine show in principle the same as the oxygen and calcium maps at a higher level of noise due to lower counting statistics. The EDX maps of fluorine, sodium and silicon exhibit bright dots along the outlines of several pores, thus these elements are enriched in the pores surfaces. The signal for sodium is faint in thin regions of the sample, possibly because of sodium evaporation under the electron beam during the measurement, while several enrichments imaged as bright dots are retained in the thicker regions of the samples. The carbon map looks similar to the oxygen and the calcium map despite the contrast between the locations of the pores and the calcite. We calculated a ratio image. Here, the pores light up meaning that the ratio of carbon content to oxygen content along the viewing direction is higher for the pore region, indicating that the pores are filled with carbon containing material (Fig. 7).

Another feature is visible in the sulfur map. Here, bright dots caused by local sulfur enrichment are present, which are not located at the positions of the pores. Between these bright dots, faint white lines are visible, interconnecting these dots to a mesh with an open size of approximately $50 \mathrm{~nm}$ (Fig. 7). This mesh size is very similar to the mesh size of the defect lines as well as the stressed domains in the two beam images. Additionally, the enrichment of sulfur visible as bright dots in Fig. 7 resembles the counterpart to the bright dots visible in Fig. 5a.

\section{Discussion}

We observed nanometer-sized pores in a spine of Holopneustes porossisimus. Due to the fact that we observed the pores in at least two spines of different individuals, prepared by different preparation techniques and as we were able to detect a pore filling containing elements not present in our preparation material, we can rule out that the pores are a preparation artifact. Comparable structures have been observed by Su, Kamat and Heuer (2000) in Heterocentrotus trigonarius, who attributed them to protein inclusions without presenting experimental evidence for attribution of the filling's nature. Other work carried out on non-crushed samples did not reveal such pores or inclusions in Neocrinus blakei (Blake, Peacor and Allard, 1984) or Strongylocentrotus francicanus and Strongylocentrotus purpuratus (Tsipursky and Buseck, 1993). Thus, it seems that not all sea urchin species develop such pores.

It was shown by Nickel and Henisch (1969) that gelgrown calcite crystals incorporate the silca gel network. Thus, it can be speculated that an organic framework pro- viding a matrix for the crystallization of the spine can also be incorporated, forming the observed pore structure. Inside the pores, fluorine, silicon and sodium are observed. The intracrystalline amino acids reported by Aizenberg et al. (1997) for Paracentrotus lividus do not contain these elements. At this stage, it is not clear whether these elements are residues of the crystallization process, either as a relict of a biological matrix or sheet confining a crystallization space, or if they were deposited for other purposes as improving mechanical or chemical performance of the spine or simply as a deposit of a residue.

In HAADF images, the pores appear much darker when compared to the surrounding material. Thus, they have to be filled with a material which is less dense than magnesian calcite. The ratio image of the carbon and oxygen EDX maps displays the pores as bright dots with respect to the surrounding calcite. This means that carbon based material has to be present in the pores, too. This finding is in line with the assumption of $\mathrm{Su}$, Kamat and Heuer (2000) as well as with the protein content of $0.2 \%-$ $0.7 \%$ observed by Aizenberg et al. (1997). Amino acids contain Nitrogen, which was not observed in our EDX investigations. The Nitrogen content in proteins is comparatively low in comparison to the carbon content, and it becomes difficult to identify this element besides oxygen and calcium due to the overlap of the N_K X-ray emission lines with the $\mathrm{O} \_\mathrm{K}$ and $\mathrm{Ca} \_\mathrm{L} \mathrm{X}$-ray emission lines.

Electron diffraction as well as powder X-ray diffraction proved the material to be calcite, which is the thermodynamically most stable modification. No twins or orientation variants were observed by TEM. From this, we can exclude a phase transformation from aragonite (or even vaterite) to calcite due to mechanical stress during the preparation procedure.

The contrast effects in the magnesian calcite are rather complicated in the micrographs recorded under two-beam conditions. There is lattice tension around some of the pores causing a characteristic peanut-shaped contrast (Fig. 4b). Linear structures, as they are caused by dislocations were visible under all imaging conditions (Figs. 4 and 5), but their contrast appeared less intense when $g=(0006)$ or $g=(01 \overline{1} 8)$ were used as operating reflections. For the images taken with $g=(0006)$, we applied contrast enhancement, in opposition to the images exhibiting high overall contrast (Figs. 4a, 5b), where these linear structures are partially obscured by the strong bright and dark patterning. Thus, these structures running perpendicular to $g=(0006)$ in Figs. $4 \mathrm{~b}$ and $5 \mathrm{a}$ can be regarded to exhibit parasitic or rest contrast. Such a finding in deformed inorganic calcite has been attributed to the decoration of the dislocation cores with beam induced point defects (Barber et al. 2007). In the sea urchin samples investigated here, one can speculate that the decoration is not due to beam damage only or mixed screw and edge character of the dislocations, but segregation of the additional elements like sodium, magnesium or sulfur to these lines, which would lead to similar contrast effect as they would cause comparable lattice deformations.

Dislocations have not been observed so far in sea urchin spines. This is not contradictory to our results. $\mathrm{Su}, \mathrm{Ka}-$ mat and Heuer (2000) prepared their samples as transver- 
sal sections of the spines. Dislocations with the proposed burgers vector $b=1 / 3\langle 11 \overline{2} 0\rangle$ would have been invisible or very low in contrast along this viewing direction. Blake, Peacor and Allard (1984) as well as Tsipursky and Buseck (1993) investigated other species which did not contain pores. We frequently observed that the dislocation lines end at the pointed edges of the pores. One can speculate that these dislocations are defects to accommodate lattice misfits during the assembly of the calcite around the pores.

Blake, Peacor and Allard (1984) observed isolated low angle boundaries and misaligned building blocks by HRTEM. Additionally, Tsipursky and Buseck (1993) observed isolated low angle boundaries.

Such boundaries are also present in the spine of Holopneustes porossisimus. While most of these boundaries tend to appear as lines due to the extinction distances, they exhibit fringe contrast in some orientation (Fig. 5b). On other micrographs, they appear as a net of faint interconnected bright lines (Fig. 5a, square). Such a net is also present in the EDX map of sulfur (Fig. 7). Impurities grown into a crystal lattice need for a lattice deformation, according to different size and coordination spheres. This has to be the case for sulfur, leading to a pattern due to lattice stress and similar to the sulfur meshes in some of the two beam images (Figs. $4 \mathrm{a}$ and $5 \mathrm{~b}$ ). In all cases, the mesh wide of the pattern is about $50 \mathrm{~nm}$.

Mosaic crystallization is common for calcium carbonate, as pointed out by Cölfen and Antonietti (2005). Sea urchin spines were found to crystallize from amorphous calcium carbonate (ACC) as observed by Politi et al. (2004). The ACC is attached as small particles of $50 \mathrm{~nm}$ in diameter. The coherence length measurements (Berman, et al. 1990, Aizenberg et al., 1997) and the nanocrystalline fracture surfaces (Nissen, 1969, Towe, 1967, O'Neill, 1981, Oaki and Imai, 2006, Oaki et al., 2006) can be attributed to this growth mechanism.

Comparison of the growth mechanism as observed by Politi et al. (2004) with the meshwork observed in diffraction contrast images as well as in the EDX maps of the spine leads to the conclusion that all the observed imperfections arise from impurities grown into the material and the resulting mismatches to accommodate the calcite lattice. The observed defect and sulfur decoration meshwork directly images the mesocrystalline substructure in the solid body of the spine.

\section{Conclusions}

The spines of Holopneustes porossisimus are mosaic single crystals. They contain pores filled with carbon, silicon, fluorine and sodium containing materials. The magnesian calcite between these pores exhibits pronounced lattice strain. It contains dislocations, partially connecting pores. Additional boundaries, probably rotational and low-angle boundaries, are present leading to a defect network. This network is mirrored by the distribution pattern of sulfur inside the magnesian calcite. We regard this pattern as a residue from the growth process of the spine by attaching and crystallizing ACC. For Holopneustes porossisimus, the mesh size of this network is about $50 \mathrm{~nm}$.
Acknowledgements. Many thanks to EDAX inc. for making available the Apollo XLT SSD-detector and TEAM software as well as to Dr. Michaela Schleifer, Dr. Felix Reinauer and Tobias Lorenz for the help with that system. We gratefully acknowledge the Zoologisch Staatssammlung München for the sea urchin spines used in this study.

\section{References}

J. Aizenberg, J. Hanson, T. F. Koetzle, S. Weiner, S. Addadi, Control of macromolecule distribution within synthetic and biogenic single calcite crystals. J. Am. Chem. Soc., 1997, 119, 881-886.

D. J. Barber, H.-R. Wenk, J. Gomez-Barreiro, E. Rybacki, G. Dresen, Basal slip and texture development in calcite: new results from torsion experiments. Phys. Chem. Minerals, 2007, 34, 7384.

A. Berman, L. Addadi, A. Kvick, L. Leiserowitz, M. Nelson, S. Weiner, Intercalation of sea-urchin proteins in calcite - study of a crystalline composite-material. Science, 1990, 250, 664-667.

F. D. Blake, D. R. Peacor, L. F. Allard, Ultrastructural and microanalytical results from echinoderm calcite: Implications for biomineralization and diagenesis of skeletal material. Micron and Microscopica Acta, 1984, 15, 85-90.

H. Cölfen, M. Antonietti, Mesokristalle: anorganische Überstrukturen durch hochparallele Kristallisation und kontrollierte Ausrichtung, Angewandte Chemie, 2005, 117, 5714-5730.

P. B. Hirsch, A. Howie, R. B. Nicholson, D. W. Pashley, M. J. Whelan, Electron microscopy oft thin crystals, Butterworth, London (1965).

A. Goetz, E. Griesshaber, W. W. Schmahl, An easy approach to increase the precision of EBSD analysis - examples from a sea urchin calcite study. Sol. State Phen., 2010, 160, 229-234.

N. Hutchinson, T. Hunt, L. Morris, Seagrass and Reef Program for Port Phillip Bay: Temperate Reefs Literature Review, Fisheries Victoria Technical Report No. 11, Department of Primary Industries, Queenscliff, Victoria, Australia (2010).

K. Kelm, E. Griesshaber, Andreé, Götz, W. W. Schmahl, How perfect are sea urchin spine single crystals? Examples from the spine of the sea urchin Holopneustes porosissimus. In: MC 2011 Kiel Proceedings Vol. 2, (Ed. W. Jäger et. al.), L-723, German Society for Electron Microscopy e.V., Kiel, 2011.

C. Moureaux, A. Pérez-Huerta, P. Compère, W. Zhu, T. Leloup, M. Cusack, P. Dubois, Strucure, composition and mechanical relations to function in sea urchin spine. J. Struct. Biol., 2010, 170, 41-49.

H. J. Nickl, H. K. Henisch, Growth of Calcite Crystals in Gels. Journal of the Electrochemical Society, 1969, 116, 1258-1260.

H.-U. Nissen, Crystal orientation and plate structure in echinoid skeletal units. Science, 1969, 166, 1150-1152.

P. L. O'Neill, Polycrystalline echinoderm calcite and its fracture mechanism. Science, 1981, 213, 646-648.

Oaki, Y, Imai, H.: Nanoengineering in echinoderms: The emergence of morphology from nanobricks. Small, 2006, 2, 66-70.

Y. Oaki, A. Kotachi, T. Miura, H. Imai, Bridged nanocrystals in biominerals and their biomimetics: Classical yet modern crystal growth on the nanoscale. Adv. Funct. Mater., 2006, 16, 16331639.

Y. Politi, T. Arad, E. Klein, S. Weiner, L. Addadi, Sea urchin spine calcite via a transient amorphous calcium carbonate phase. Science, 2004, 306, 1164-1164.

D. M. Raupp, The Endoskeleton, In: Physiology of Echinodermata (Ed. R. A. Boolootián) pp. 375-395. Interscience, (New York) 1966.

W. J. Schmidt, Die Bausteine des Tierkörpers in Polarisiertem Lichte, Verlag F. Cohen, Bonn, 1924.

W. J. Schmidt, Die Sklettstücke der Stachelhäuter als Biokristalle. Zool. Jb. Allgem. Zoologie, 1930, 47, 357-510.

I. Sethmann, G. Wörheide, Structure and composition of calcareous sponge spicules: A review and comparison to structurally related biominerals. Micron, 2008, 39, 209-228.

X. Su, S. Kamat, A. H. Heuer, The structure of sea urchin spines, large biogenic single crystals. J. Mater. Sci., 2000, 35, 55455551.

J. Titschack, F. Goetz-Neunhoeffer, J. Neubauer, Magnesium quantification in calcites $\left[(\mathrm{Ca}, \mathrm{Mg}) \mathrm{CO}_{3}\right]$ by Rietveld-based XRD analy- 
sis: Revisiting a well-established method. American Mineralogist, 2011, 96, 1028-1038.

K. M. Towe, Echinoderm calcite: single crystal or polycrystalline aggregate. Science, 1967, 157, 1048-1050.

D. F. Travis, The comparative ultrastructure and organization of fife calcified tissues, In: Bilogical Calcification: Cellular and Molecular Aspects (Ed. H. Schraer), pp. 203-311, Appelton-CenturyCrofts, (New York) 1970.

S. J. Tsipursky, P. R. Buseck, Structure of magnesian calcite from sea urchins. Am. Mineral. 1993, 78, 775-781.
V. von Ebner, Über den feineren Bau der Sklettheile der Kalkschwämme nebst Bemerkungen über Kalkskelette überhaupt. Sitzungsberichte der Kaiserlichen Akademie der Wissenschaften, 1. Abteilung, 1887, 95, 55-149.

S. Weiner, Organic matrix like macromolecules associated with the mineral phase of sea urchin skeletal plates and teeth. J. Exp. Zool., 1985, 234, 7-15. 